\title{
An Inequality for Second Order Differential Equation with Retarded Argument
}

\author{
Erdoğan Şen \\ Department of Mathematics, Namık Kemal University, Tekirdă̆, Turkey \\ E-mail:esen@nku.edu.tr \\ Received April 23, 2011; revised May 10, 2011; accepted May 20, 2011
}

\begin{abstract}
Applications of differential equations with retarded argument can be encountered in the theory of automatic control, in the theory of self-oscillatory systems, in the study of problems connected with combustion in rocket engines, in a number of problems in economics, biophysics. The problems in this areas can be solved reducing differential equations with retarded argument. In this work an important inequality for second order differential equation with retarded argument is obtained.
\end{abstract}

Keywords: Differential Equation with Retarded Argument, Inequality

\section{Introduction}

In this study we consider the equation

$$
L(w)=w^{\prime \prime}(t)+\lambda w(t)+M(t) w(t-\Delta(t))=0
$$

on an interval $I$. Where $\lambda$ is a real parameter; $M(t)$ and $\Delta(t)$ are continuous functions on $I ; t-\Delta(t) \geq 0$ and $1 \geq \Delta(t)>0$ for each $t \in I$.

\section{An Inequality for Second Order Differential Equation with Retarded Argument}

Theorem. Let us denote by every point with $t_{0}$ which is satisfying the mean-value theorem for a continuous solution $w\left(t_{j}\right)$ of (1) on $\left[t_{j}-\Delta(t), t_{j}\right] \subseteq I$ for each $t_{j} \in I$ and $j \in J$, where $J$ is an index set. Also let us assume that $\sup M(t)=M_{0}$ where $M_{0}$ is a real number. Then for all $t_{j}$ in the equation

$$
\left\|w\left(t_{0_{j}}\right)\right\| \mathrm{e}^{-k\left|t_{j}-t_{0_{j}}\right|} \leq\left\|w\left(t_{j}\right)\right\| \leq\left\|w\left(t_{0_{j}}\right)\right\| \mathrm{e}^{k\left|t_{j}-t_{0_{j}}\right|}
$$

where

$$
\left\|w\left(t_{j}\right)\right\|=\left[\left|w\left(t_{j}\right)\right|^{2}+\left|w^{\prime}\left(t_{0_{j}}\right)\right|^{2}\right]^{1 / 2}, k=1+|\lambda|+\frac{3}{2}\left|M_{0}\right|
$$

Proof. From the mean-value theorem we can write the followings:

$$
\frac{w\left(t_{j}\right)-w\left(t_{j}-\Delta\left(t_{j}\right)\right)}{\Delta\left(t_{j}\right)}=w^{\prime}\left(t_{0_{j}}\right)
$$

$$
w\left(t_{j}-\Delta\left(t_{j}\right)\right)=w\left(t_{j}\right)-w^{\prime}\left(t_{0_{j}}\right) \Delta\left(t_{j}\right)
$$

and

$$
\left|w\left(t_{j}-\Delta\left(t_{j}\right)\right)\right| \leq\left|w\left(t_{j}\right)\right|+\left|w^{\prime}\left(t_{0_{j}}\right)\right|
$$

Now we let $u\left(t_{j}\right)=\left\|w\left(t_{j}\right)\right\|^{2}$. Thus

$$
u=\bar{w}+w^{\prime} \overline{w^{\prime}}
$$

where $\bar{w}\left(t_{j}\right)=\overline{w\left(t_{j}\right)}$. Then

$$
u^{\prime}=w^{\prime} \bar{w}+w \overline{w^{\prime}}+w^{\prime \prime} \overline{w^{\prime}}+w^{\prime} \overline{w^{\prime \prime}}
$$

From the definition of a derivative it follows that $\overline{w^{\prime}}=\bar{w}^{\prime}$. Also $\left|w\left(t_{j}\right)\right|=\left|\overline{w\left(t_{j}\right)}\right|$. Therefore

$$
\left|u^{\prime}\left(t_{j}\right)\right| \leq 2\left|w\left(t_{j}\right)\right|\left|w^{\prime}\left(t_{0_{j}}\right)\right|+2\left|w^{\prime}\left(t_{0_{j}}\right)\right|\left|w^{\prime \prime}\left(t_{j}\right)\right|
$$

Since $w$ satisfies $L(w)=0$ we have

$$
w^{\prime \prime}\left(t_{j}\right)=-\lambda w\left(t_{j}\right)-M\left(t_{j}\right) w\left(t_{j}-\Delta\left(t_{j}\right)\right)
$$

and hence applying (3)

$$
\begin{aligned}
\left|w^{\prime \prime}\left(t_{j}\right)\right| & \leq|\lambda|\left|w\left(t_{j}\right)\right|+\left|M_{0}\right|\left|w\left(t_{j}-\Delta\left(t_{j}\right)\right)\right| \\
& \leq|\lambda|\left|w\left(t_{j}\right)\right|+\left|M_{0}\right|\left(\left|w\left(t_{j}\right)\right|+\left|w^{\prime}\left(t_{0_{j}}\right)\right|\right)
\end{aligned}
$$


Using (5) in (4) we obtain

$$
\begin{aligned}
\left|u^{\prime}\left(t_{j}\right)\right| \leq 2\left|w\left(t_{j}\right)\right|\left|w^{\prime}\left(t_{0_{j}}\right)\right|+2\left|w^{\prime}\left(t_{0_{j}}\right)\right| \\
\cdot\left\{|\lambda|\left|w\left(t_{j}\right)\right|+\left|M_{0}\right|\left(\left|w\left(t_{j}\right)\right|+\left|w^{\prime}\left(t_{0_{j}}\right)\right|\right)\right\} \\
=2\left(1+|\lambda|+\left|M_{0}\right|\right)\left|w\left(t_{j}\right)\right|\left|w^{\prime}\left(t_{0_{j}}\right)\right|+2\left|M_{0}\right|\left|w^{\prime}\left(t_{0_{j}}\right)\right|^{2}
\end{aligned}
$$

Now applying the fact

$$
2\left|w\left(t_{j}\right)\right|\left|w^{\prime}\left(t_{0_{j}}\right)\right| \leq\left|w\left(t_{j}\right)\right|^{2}+\left|w^{\prime}\left(t_{0_{j}}\right)\right|^{2}
$$

we get

$$
\begin{aligned}
\left|u^{\prime}\left(t_{j}\right)\right| & \leq\left(1+|\lambda|+\left|M_{0}\right|\right)\left|w\left(t_{j}\right)\right|^{2} \\
& +\left(1+|\lambda|+3\left|M_{0}\right|\right)\left|w^{\prime}\left(t_{0_{j}}\right)\right|^{2} \\
& \leq 2\left(1+|\lambda|+\frac{3}{2}\left|M_{0}\right|\right)\left(\left|w\left(t_{j}\right)\right|^{2}+\left|w^{\prime}\left(t_{0_{j}}\right)\right|^{2}\right)
\end{aligned}
$$

or

$$
\left|u^{\prime}\left(t_{j}\right)\right| \leq 2 k u\left(t_{j}\right)
$$

This is equivalent to

$$
-2 k u\left(t_{j}\right) \leq u^{\prime}\left(t_{j}\right) \leq 2 k u\left(t_{j}\right)
$$

And these inequalities lead directly to (2). Indeed con- sider the right inequality which can be written as $u^{\prime}-2 k u \leq 0$. It is equivalent to

$$
\mathrm{e}^{-2 k t_{j}}\left(u^{\prime}-2 k u\right)=\left(\mathrm{e}^{-2 k t_{j}} u\right)^{\prime} \leq 0
$$

If $t_{j}>t_{0_{j}}$ we integrate from $t_{0}$ to $t_{0_{j}}$ obtaining

$$
\mathrm{e}^{-2 k t_{j}} u\left(t_{j}\right)-\mathrm{e}^{-2 k t_{0_{j}}} u\left(t_{0_{j}}\right) \leq 0
$$

or

$$
u\left(t_{j}\right) \leq u\left(t_{0_{j}}\right) \mathrm{e}^{2 k\left(t_{j}-t_{0_{j}}\right)}
$$

The left inequality in (6) similarly implies

$$
\left\|w\left(t_{0_{j}}\right)\right\| \mathrm{e}^{-k\left(t_{j}-t_{0_{j}}\right)} \leq\left\|w\left(t_{j}\right)\right\|, \quad t_{j}>t_{0_{j}}
$$

and therefore

$$
\left\|w\left(t_{0_{j}}\right)\right\| \mathrm{e}^{-k\left|t_{j}-t_{0_{j}}\right|} \leq\left\|w\left(t_{j}\right)\right\| \leq\left\|w\left(t_{0_{j}}\right)\right\| \mathrm{e}^{k\left|t_{j}-t_{0_{j}}\right|}, \quad t_{j}>t_{0_{j}}
$$

which is just (2) for $t_{j}>t_{0_{j}}$. The case $t_{j}<t_{0_{j}}$ may be considered analogically.

\section{References}

[1] E. A. Coddington and N. Levinson, "Theory of Ordinary Differential Equations," McGraw-Hill, New York, 1955.

[2] S. B. Norkin, "Differential Equations of the Second Order with Retarded Argument," AMS, Providence, 1972. 